BOSQUE 25(3): 25-32, 2004

\title{
Efecto del cambio de las condiciones de maquinado sobre la rugosidad superficial de Pinus radiata D. Don
}

Effect of a change in machining conditions on surface roughness of Pinus radiata, D. Don

\author{
ALFREDO AGUILERA, MARIO VEGA
}

Instituto de Tecnología de Productos Forestales, Universidad Austral de Chile, Casilla 567,

Valdivia, Chile. E-mail: aguilera@uach.cl

\section{SUMMARY}

The effect of the machining conditions on the resulting surface roughness during the milling process of radiata pine was studied. The consumption of power whilst cutting was monitored, and in addition the angle of the fiber of the test specimens was varied to observe the effect on the surface quality. The results contribute to the knowledge of the machining conditions for this species and are of great commercial interest. The influence of both the feed and rotation speed on the product quality are clear. The relationship of these speeds, considered with the depth of cut and the cutting tool diameter (constants in this case), permit a definition of chip thickness as a key factor in the determination of quality and power consumption in the process. The values relating to the angle of fibers confirm that an increase in the angle causes a pronounced deterioration in the quality, and that this correlated well with the chip thickness.

Key words: machining, surface roughness, radiata.

\section{RESUMEN}

Se estudia en el fresado de pino radiata, el efecto de las condiciones de maquinado sobre la rugosidad superficial resultante del proceso. Al mismo tiempo se monitorea el consumo de potencia de corte y adicionalmente se hacen variar los ángulos de las fibras de las probetas para observar su efecto sobre la calidad superficial. Los resultados permiten contribuir al conocimiento de las condiciones de maquinado para esta especie de gran interés comercial. Se observan claramente las influencias de las velocidades de avance y rotación sobre la calidad. Se indica que la relación de estas velocidades más la profundidad de corte y el diámetro de la herramienta de corte (los cuales permanecieron constantes) permiten definir el espesor de viruta, como elemento clave en la determinación tanto de la calidad como del consumo de potencia del proceso. En cuanto a la influencia del ángulo de las fibras, los valores encontrados permiten confirmar la teoría, en cuanto que un incremento del ángulo deteriora fuertemente la calidad, obteniéndose muy buenas correlaciones respecto del espesor de viruta.

Palabras clave: maquinado, superficie, rugosidad, radiata.

\section{INTRODUCCION}

La industria de la remanufactura del pino radiata en el país muestra tendencias interesantes, ya que cada vez aquellas plantas industriales dedicadas solamente a la producción y exportación de productos primarios están incorporando la tecnología que permite generar productos cuyo valor agregado y cuyos precios son mayores.
Es así como estos productos, madera seca dimensionada (MSDi), es decir, madera estable dimensionalmente por su bajo contenido de humedad y cepillada por sus cuatro caras y molduras decorativas principalmente, presentan volúmenes $\left(\mathrm{m}^{3}\right)$ y montos (US\$) exportados que cada año van superándose, lo cual refleja la interesante demanda por estos productos, todos producidos con madera de pino radiata, demanda que tiene su origen en los 
BOSQUE 25(3): 25-32, 2004

Efecto del cambio de las condiciones de maquinado sobre la rugosidad superficial de Pinus radiata D. Don

mercados de América del Norte y proporciones interesantes en el mercado nacional.

Para el caso de las molduras sólidas de pino radiata, la poblemática está asociada en aspectos tales como, la aplicación de pintura, la aplicación de yeso u otros productos de terminación. Por otra parte, otro problema no menos importante y relevante sobre los primeros mencionados, es el desconocimiento de cuáles deben ser las condiciones en las que debe trabajar una máquina de manera tal que la rugosidad superficial de la madera permita que los recubrimientos se adhieran eficientemente sobre la nueva superficie. Además, el ajuste inadecuado de las condiciones de maquinado, ya sea en cepilladora o moldurera, además de generar una calidad superficial inapropiada, desgastará inútilmente las herramientas de corte, con un consumo de energía excesivo.

Esta calidad superficial inapropiada se presenta en dos extremos, el extremo negativo en el cual la calidad de la superficie es notoriamente deficiente, es decir, con levantamientos de fibras y ondulaciones pronunciadas, y en el otro extremo, una calidad en exceso buena, es decir, una superficie extremadamente pulida con ondulaciones imperceptibles. Esta última condición es la que genera el mayor desgaste de herramientas y el mayor consumo de potencia, además que la calidad del sustrato no es el más adecuado para sostener un recubrimiento determinado.

Siendo el problema fundamental de la industria de la madera el desconocimiento de las condiciones de corte para lograr un maquinado correcto, donde los problemas de calidad no siempre están solamente asociados a la calidad de la materia prima sino que también y de una manera importante a procesos mal planificados y ejecutados.

La importancia de controlar el estado superficial de la madera lo indica Lemaster y Dornfeld (1), señalando que la rugosidad superficial va a estar directamente relacionada con el uso que se le quiera dar a una pieza, además, un control efectivo de la calidad de la superficie permite asegurar un ensamblaje correcto de las piezas y determina la aplicación de adhesivos y productos de terminación.

En estudios anteriores de maquinado de MDF y especies europeas Aguilera et al. (2-4) encontraron una clara relación entre el espesor de viruta y la rugosidad superficial, con buenos niveles de correla- ción, mostrando tendencias lineales positivas, donde las mejores calidades fueron obtenidas con espesores de viruta pequeños, sin embargo con altos consumos de energía y con una disminución de la vida útil de la herramienta de corte.

El desgaste de las herramientas de corte está relacionado con la calidad de las superficies. Según Lemaster et al. (5) al hacer variar las velocidades de rotación, la velocidad de avance y el modo de corte (oposición o concordancia), las mayores velocidades de corte (o rotación) generarán una mejor calidad superficial, sin embargo, el modo de corte también debe ser considerado, ya que éste va a determinar que una superficie se degrade más por un efecto sobre el desgaste acelerado de la arista de corte.

En relación a los consumos de energía y potencia, los estudios realizados por otros autores $(6,7)$ indican que para una condición de corte dada, cuyo resultado genera un espesor de viruta que fluctúa entre determinados rangos, se estará en una zona de bajo consumo, sin embargo, si el espesor de viruta se encuentra fuera de esta zona, es decir, con valores del espesor de viruta muy pequeño o muy grande, los consumos de energía y por tanto los requerimientos de potencia de corte aumentarán de una manera exponencial.

En lo que respecta a la influencia de los ángulos de las fibras sobre la calidad superficial y el consumo de energía, se encontró que las señales de energía se correlacionan muy bien con las características del proceso de maquinado y al mismo tiempo con la calidad superficial resultante. De esta manera, las superficies más degradadas generan señales más fuertes ocurriendo lo inverso con las superficies más lisas. Además, para una condición de corte dada, el cambio del ángulo de las fibras afectó sensiblemente las energías producidas durante el proceso (8).

El objetivo principal de este estudio es el de evaluar la calidad del maquinado de pino radiata. Como objetivos secundarios se determinarán cómo influyen los cambios de las condiciones de maquinado sobre la rugosidad superficial, y sobre el consumo de potencia. Se buscará establecer relaciones entre las condiciones de maquinado y la rugosidad resultante y finalmente se estudiará el efecto del cambio de los ángulos de las fibras sobre la calidad y el consumo de energía. 
BOSQUE 25(3): 25-32, 2004 Efecto del cambio de las condiciones de maquinado sobre la rugosidad superficial de Pinus radiata D. Don

\section{MATERIAL Y METODOS}

Para realizar los ensayos se consideraron los materiales siguientes: (a) La madera es pino radiata de densidad y humedad constante (cuadro 1), se consideró en la zona de corte de cada probeta un número constante de anillos de crecimiento (figura 1). (b) Las probetas: se trabaja con dos tipos diferentes de probetas. Una probeta pequeña de $120 \mathrm{~mm}$ de largo y $35 \mathrm{~mm}$ de ancho, la cual se utiliza para los ensayos donde se mide la rugosidad superficial, y una probeta de $1.000 \mathrm{~mm}$ de largo y $35 \mathrm{~mm}$ de ancho, la que se usa para las mediciones de potencia de corte dado que la curva de estabilización de la potencia se alcanza con un largo mínimo de la probeta superior a $120 \mathrm{~mm}$. (c) Una máquina tupí con motor de $4 \mathrm{~kW}$ de potencia máxima, velocidad de rotación del eje de entre 3.200 y 8.000 r/min y un carro de avance automático con 4 velocidades de alimentación. (d) Un rugosímetro de contacto tipo stylus modelo Mitutoyo SJ-210. (e) Un Wattímetro numérico conectado a computador para medir y registrar la potencia de corte. (f) Herramientas de corte: 2 cuchillos en el cabezal. El cabezal posee las siguientes dimensiones D: 122, B: 60, d: 40 mm, los cuchillos son de carburo de tungsteno de $60 \times 30 \times 3$ $\mathrm{mm}$ con perfil recto cuya geometría es $\alpha=17^{\circ}$, $\beta=45,5^{\circ}$ y $\gamma=27,5^{\circ}$, siendo $\alpha$ el ángulo de incidencia, $\beta$ el ángulo de perfil y $\gamma$ el ángulo de ataque.
Para la selección de las condiciones de maquinado se tomará como base las velocidades de rotación y de avance, lo cual generará niveles variables de los espesores de viruta, estos se determinan considerando la fórmula:

$$
e c=\left(\frac{V f}{Z * N}\right) * \sqrt{a p / D}(\mathrm{~mm})
$$

Donde

ec $=$ espesor de viruta $(\mathrm{mm})$

$\mathrm{N}=$ velocidad de rotación $\left(\mathrm{min}^{-1}\right)$

$\mathrm{Vf}=$ velocidad de alimentación $(\mathrm{m} / \mathrm{min})$

ap $=$ profundidad de corte $(\mathrm{mm})$

$\mathrm{Z}=$ número de cuchillos

$\mathrm{D}$ = diámetro del cabezal $(\mathrm{mm})$

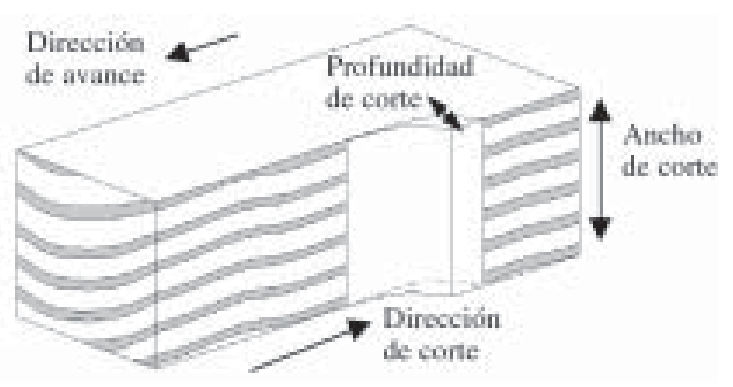

Figura 1. Características generales de las probetas de ensayos.

General characteristics of the test specimens.

CUADRO 1

Descripción de parámetros de maquinado.

Description of the machining parameters.

\begin{tabular}{|l|c|l|l|l|l|l|}
\hline $\begin{array}{l}\text { Parámetros } \\
\text { de acción }\end{array}$ & Nivel & Unidad & $\begin{array}{l}\text { Parámetros } \\
\text { de medición }\end{array}$ & Unidad & Constantes & Nivel \\
\hline $\begin{array}{l}\text { Velocidad } \\
\text { de rotación }\end{array}$ & $\begin{array}{l}3.200,4.200, \\
6.000\end{array}$ & $\mathrm{r} / \mathrm{min}$ & $\begin{array}{l}\text { Rugosidad } \\
\text { superficial } \mathrm{Rz}\end{array}$ & $\mu \mathrm{m}$ & $\begin{array}{l}\text { Ancho } \\
\text { de corte }\end{array}$ & $35 \mathrm{~mm}$ \\
\hline $\begin{array}{l}\text { Velocidad } \\
\text { de avance }\end{array}$ & $4,8,11,22$ & $\mathrm{~m} / \mathrm{min}$ & $\begin{array}{l}\text { Potencia } \\
\text { de corte }\end{array}$ & $\mathrm{kW}$ & $\begin{array}{l}\text { Profundidad } \\
\text { de corte }\end{array}$ & $2 \mathrm{~mm}$ \\
\hline $\begin{array}{l}\text { Angulo } \\
\text { de las fibras }\end{array}$ & $0,30,60$ & grados & & \multirow{2}{*}{$\begin{array}{l}\text { Modo de } \\
\text { corte }\end{array}$} & Oposición \\
\hline
\end{tabular}


BOSQUE 25(3): 25-32, 2004

Efecto del cambio de las condiciones de maquinado sobre la rugosidad superficial de Pinus radiata D. Don

Con la combinación de ambas velocidades, de rotación y de avance, y el espesor de viruta resultante, se analizan 12 tratamientos diferentes, con 4 repeticiones cada uno (cuadro 2). Para cada tratamiento se mide la rugosidad superficial utilizando el parámetro "Rz", el cual representa la media de los valores absolutos de las alturas de las cinco crestas del perfil más altas y de las profundidades de los cinco valles del perfil más bajos dentro de una longitud dada (figura 2).

En forma paralela, se repiten los tratamientos considerando una variación del ángulo de las fibras, de manera de evaluar el cambio de rugosidad asociado a esta variación.

Adicionalmente se mide la potencia de corte con un watímetro numérico, pero dada la velocidad li-

CUADRO 2

Descripción de los tratamientos. Description of treatment.

\begin{tabular}{|l|c|c|c|c|c|c|c|}
\hline \multicolumn{9}{|c}{} & \multicolumn{4}{c|}{$\begin{array}{c}\text { Rugosidad Rz según } \\
\text { ángulo de las fibras }\end{array}$} \\
\hline $\mathrm{N}$ & Vf & ec & Repeticiones & $0^{\circ}$ & $30^{\circ}$ & $60^{\circ}$ & Pc en $0^{\circ}$ \\
\hline 3.200 & 4 & 0,08 & 4 & $\checkmark$ & $\checkmark$ & $\checkmark$ & $\checkmark$ \\
4.200 & 4 & 0,06 & 4 & $\checkmark$ & $\checkmark$ & $\checkmark$ & $\checkmark$ \\
6.000 & 4 & 0,04 & 4 & $\checkmark$ & $\checkmark$ & $\checkmark$ & $\checkmark$ \\
3.200 & 8 & 0,16 & 4 & $\checkmark$ & $\checkmark$ & $\checkmark$ & $\checkmark$ \\
4.200 & 8 & 0,12 & 4 & $\checkmark$ & $\checkmark$ & $\checkmark$ & $\checkmark$ \\
6.000 & 8 & 0,09 & 4 & $\checkmark$ & $\checkmark$ & $\checkmark$ & $\checkmark$ \\
3.200 & 11 & 0,22 & 4 & $\checkmark$ & $\checkmark$ & $\checkmark$ & $\checkmark$ \\
4.200 & 11 & 0,17 & 4 & $\checkmark$ & $\checkmark$ & $\checkmark$ & $\checkmark$ \\
6.000 & 11 & 0,12 & 4 & $\checkmark$ & $\checkmark$ & $\checkmark$ & $\checkmark$ \\
3.200 & 22 & 0,44 & 4 & $\checkmark$ & $\checkmark$ & $\checkmark$ & $\checkmark$ \\
4.200 & 22 & 0,34 & 4 & $\checkmark$ & $\checkmark$ & $\checkmark$ & $\checkmark$ \\
6.000 & 22 & 0,24 & 4 & $\checkmark$ & $\checkmark$ & $\checkmark$ & $\checkmark$ \\
\hline
\end{tabular}

Donde:
$\mathrm{N}=$ velocidad de rotación $\left(\mathrm{min}^{-1}\right)$
$\mathrm{Rz}=\operatorname{rugosidad}(\mu \mathrm{m})$
$\mathrm{Vf}=$ velocidad de avance $(\mathrm{m} / \mathrm{min})$
$\mathrm{Pc}=$ potencia de corte $(\mathrm{kW})$

ec $=$ espesor de viruta $(\mathrm{mm})$

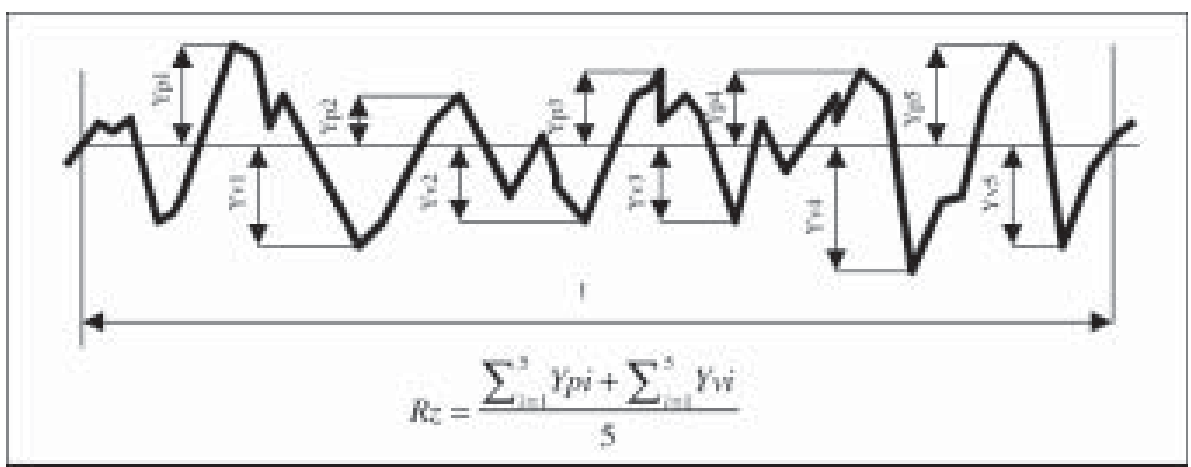

Figura 2. Parámetro Rz de rugosidad superficial.

Surface roughness Rz parameter. 
BOSQUE 25(3): 25-32, 2004

Efecto del cambio de las condiciones de maquinado sobre la rugosidad superficial de Pinus radiata D. Don

mitada en la adquisición de los datos durante el maquinado, la probeta para estos ensayos debe medir a lo menos un metro de largo. De esta manera se repiten los ensayos anteriormente descritos, sólo para la condición de madera con ángulo de las fibras de $0^{\circ}$.

\section{RESULTADOS Y DISCUSION}

Los resultados que se presentan a continuación son consistentes con los antecedentes bibliográficos en cuanto que el espesor de viruta es un buen indicador de la calidad superficial resultante de los procesos de maquinado. Al considerar en el análisis el cambio en los tres niveles del ángulo de inserción de las fibras queda aún de manifiesto el efecto de la variación de los parámetros de corte, sobre la rugosidad superficial. El cuadro 3 muestra los resultados obtenidos para rugosidad superficial y potencia de corte, según las diferentes condiciones de maquinado y ángulo de las fibras.

En este cuadro es posible identificar claramente el efecto del aumento de la velocidad de avance Vf sobre el deterioro de la calidad de la superficie maquinada. Considerando, por ejemplo, la situación para el ángulo de $0^{\circ}$ de inserción de las fibras, el aumento gradual de la velocidad de avance para una velocidad de rotación constante indica un deterioro sostenido de la calidad superficial. Si a esto se le agrega el efecto del cambio del ángulo de las fibras, el deterioro superficial es aún mayor al incrementar los ángulos. Este análisis queda reflejado en la figura 3.

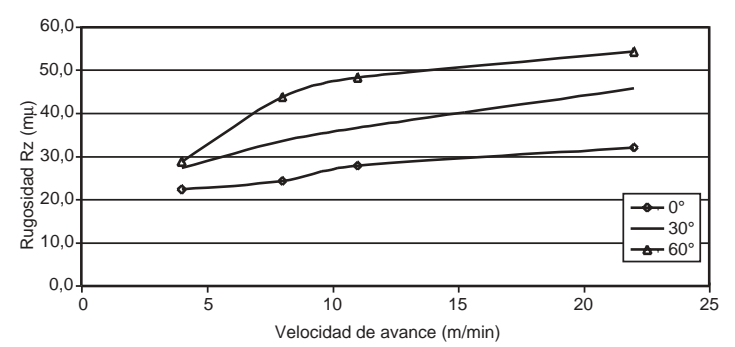

Figura 3. Influencia de la velocidad de avance y del ángulo de las fibras sobre la rugosidad superficial.

Feed speed and fiber angle influence on surface roughness.

\section{CUADRO 3}

Rugosidad superficial Rz $(\mu \mathrm{m})$ según ángulo de las fibras y espesor de viruta $(\mathrm{mm})$ y potencia de corte $(\mathrm{kW})$ para $0^{\circ}$.

Surface roughness $\mathrm{Rz}(\mu \mathrm{m})$ according to fiber angle, chip thickness ( $\mathrm{mm}$ ), and cutting power $(\mathrm{kW})$ for $0^{\circ}$

\begin{tabular}{|c|c|c|ccc|c|}
\hline \multicolumn{7}{|c|}{ Rugosidad Rz } \\
\hline $\mathrm{N}$ & Vf & ec & $0^{\circ}$ & $30^{\circ}$ & $60^{\circ}$ & Pc \\
\hline 3.200 & 4 & 0,08 & 23,36 & 28,35 & 30,65 & 0,129 \\
4.200 & 4 & 0,06 & 22,00 & 27,78 & 28,91 & 0,132 \\
6.000 & 4 & 0,04 & 21,23 & 25,69 & 26,48 & 0,165 \\
3.200 & 8 & 0,16 & 26,94 & 35,78 & 48,65 & 0,169 \\
4.200 & 8 & 0,12 & 24,00 & 32,85 & 41,06 & 0,174 \\
6.000 & 8 & 0,09 & 21,49 & 31,53 & 41,20 & 0,174 \\
3.200 & 11 & 0,22 & 27,75 & 39,54 & 61,75 & 0,184 \\
4.200 & 11 & 0,17 & 28,00 & 35,43 & 46,54 & 0,178 \\
6.000 & 11 & 0,12 & 27,37 & 34,67 & 35,94 & 0,174 \\
3.200 & 22 & 0,44 & 35,45 & 56,61 & 61,94 & 0,230 \\
4.200 & 22 & 0,34 & 32,00 & 42,76 & 58,49 & 0,227 \\
6.000 & 22 & 0,24 & 28,27 & 37,50 & 41,90 & 0,257 \\
\hline
\end{tabular}

$\mathrm{N}=$ velocidad de rotación $\left(\mathrm{min}^{-1}\right)$

$\mathrm{Rz}=$ rugosidad superficial $(\mu \mathrm{m})$

$\mathrm{Vf}=$ velocidad de avance $(\mathrm{m} / \mathrm{min})$

$\mathrm{Pc}=$ potencia de corte $(\mathrm{kW})$

ec $=$ espesor de viruta $(\mathrm{mm})$

$0^{\circ}, 30^{\circ}, 60^{\circ}=$ ángulos fibras 
BOSQUE 25(3): 25-32, 2004

Efecto del cambio de las condiciones de maquinado sobre la rugosidad superficial de Pinus radiata D. Don

En la figura 3 se puede apreciar que para pino radiata se ve un deterioro gradual de la calidad de la superficie al ir incrementando la velocidad de avance, considerando este parámetro de manera aislada, siendo para $0^{\circ}$ de ángulo de las fibras donde se encuentran los niveles inferiores de deterioro. Además, aunque una alta velocidad de avance es negativa para la calidad, el aumento del ángulo de las fibras es crítico sobre los resultados.

Si a este análisis se considera el efecto de la velocidad de rotación, se puede ver, en la figura 4 , que las tendencias de las curvas indican que frente a un aumento de estas velocidades, la rugosidad superficial disminuye. Siendo consistente con los antecedentes de la figura anterior, el incremento del ángulo de las fibras contribuye a deteriorar la superficie, independiente de la velocidad elegida.

Si bien estos parámetros, velocidad de avance y de rotación indican claramente sus efectos sobre la calidad de las superficies, al tratarse de manera aislada son elementos poco precisos de análisis; por esto es necesario referir uno en relación al otro para poder concluir sobre su efecto en la calidad de las superficies.

Entonces, si se considera la interacción de los parámetros de corte: velocidad de rotación, velocidad de avance, profundidad de corte, diámetro de la herramienta y número de cuchillos, en el análisis de los resultados obtenidos se puede entonces notar el efecto del espesor de viruta sobre la rugosidad superficial (figura 5).

De la figura podemos indicar que la variación observada en la rugosidad superficial, para el caso de $0^{\circ}$ de ángulo de las fibras, se ve influenciada fuertemente por el cambio en el espesor de viruta, mostrando una tendencia lineal con un buen índice de correlación. De esta manera, los espesores de viruta pequeños indicarán mejores estados superficiales

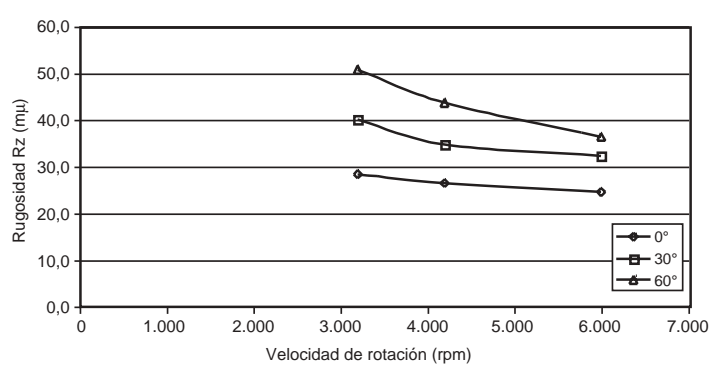

Figura 4. Influencia de la velocidad de rotación y del ángulo de las fibras sobre la rugosidad superficial. Cutting speed and fiber angle influence on surface roughness.

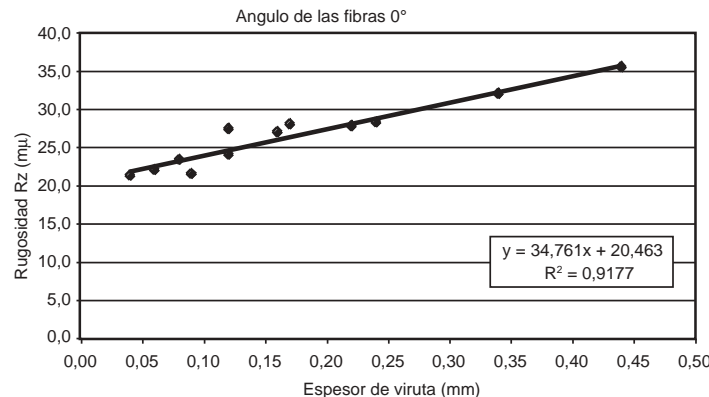

Figura 5. Influencia del espesor de viruta sobre la rugosidad superficial para $0^{\circ}$ del ángulo de las fibras.

Chip thickness influence on surface roughness at $0^{\circ}$ fiber angle.

después de realizado el maquinado de la madera de pino radiata.

Al considerar los cambios en los ángulos de las fibras en la relación espesor de viruta-rugosidad superficial, la figura 6 indica claramente los resultados obtenidos. En esta figura se aprecia un efecto neto del incremento del ángulo sobre el deterioro de las superficies, reafirmando el efecto del incremento del espesor de viruta sobre la calidad superficial. Sin embargo, para el caso del ángulo de $60^{\circ}$ se observa un deterioro más fuerte en relación a los otros ángulos para bajos espesores de viruta. Se puede indicar entonces que para las mismas condiciones de corte un cambio del ángulo que se encuentre cercano a $60^{\circ}$ o superior generará una superficie de calidad notoriamente deteriorada respecto de ángulos inferiores.

Para el caso de la potencia de corte se evaluó solamente en $0^{\circ}$ de ángulo de fibra, dado que es necesario un largo mínimo de la madera para poder adquirir los datos, lo cual es imposible en los otros ángulos. La figura 7 grafica los resultados para la relación espesor de viruta-potencia de corte.

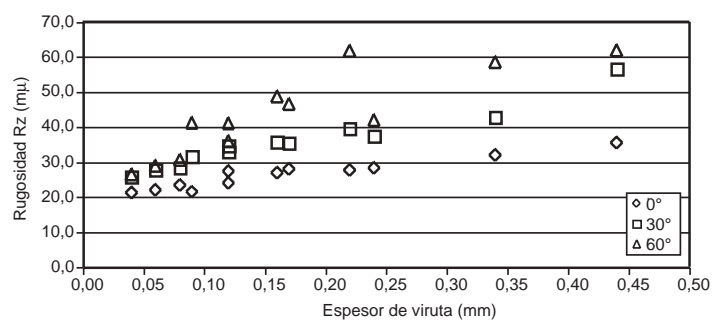

Figura 6. Influencia del espesor de viruta sobre la rugosidad superficial para diferentes ángulos de las fibras.

Chip thickness influence on surface roughness for differing fiber angles. 
BOSQUE 25(3): 25-32, 2004

Efecto del cambio de las condiciones de maquinado sobre la rugosidad superficial de Pinus radiata D. Don

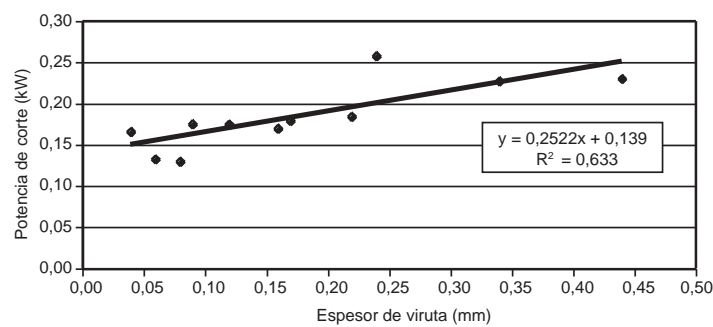

Figura 7. Relación espesor de viruta-potencia de corte. Relationship of chip thickness to cutting power.

Los resultados observados en esta figura son consistentes con los antecedentes bibliográficos, dado que un aumento del espesor de viruta está bien correlacionado con un aumento progresivo de los requerimientos de potencia de corte. Los ensayos realizados en pino radiata, según la configuración de las probetas indicadas en la figura $1 \mathrm{y}$ las condiciones dadas en el cuadro 2, permitieron establecer claramente las relaciones observadas en esta figura. De esta manera sería factible estimar a priori conociendo las condiciones de maquinado, cuál sería el consumo de potencia requerido para el proceso.

Por otra parte, se evaluó el efecto potencia de corte-rugosidad superficial, el cual se indica en la figura 8. De este gráfico se infiere que una mayor rugosidad superficial está asociada a una mayor demanda de potencia de corte, lo cual es consistente con las observaciones indicadas en relación al efecto del espesor de viruta, es decir, un espesor de viruta de mayor magnitud indica una mayor demanda de potencia y, al mismo tiempo, este mayor espesor de viruta se asocia según los resultados anteriormente expuestos a una superficie cada vez más

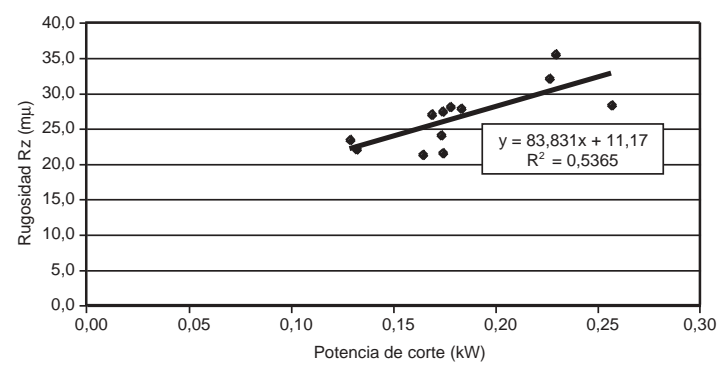

Figura 8. Relación potencia de corte-rugosidad superficial.

Relationship of cutting power to surface roughness. deteriorada. Con este resultado, al igual que el anterior, sería posible estimar la rugosidad resultante mediante un control de la potencia de corte.

Finalmente, se puede señalar que los resultados obtenidos son satisfactorios, ya que concuerdan con la bibliografía consultada y permiten ser un aporte en el estudio de los procesos de maquinado del pino radiata creciendo en Chile.

\section{CONCLUSIONES}

- Tanto las velocidades de avance como de rotación muestran comportamientos consistentes en cuanto a su efecto sobre la rugosidad superficial, donde un aumento de la velocidad de avance genera superficies de inferior calidad para velocidades de rotación constantes. De otra manera, un aumento de la velocidad de rotación produce superficies menos rugosas para velocidades de avance constantes.

- Independiente de las condiciones de maquinado anteriormente expuestas y del espesor de viruta, un cambio en el ángulo de las fibras de la madera tiene un gran efecto sobre la calidad de las superficies.

- El espesor de viruta es un buen indicador de la rugosidad superficial para diferentes condiciones de maquinado, ya que integra una serie de condiciones de corte en conjunto.

- El espesor de viruta mostró ser un buen indicador de los requerimientos de potencia para los diferentes ensayos en pino radiata. Es posible estimar la potencia de corte con una determinación del espesor de viruta.

- La potencia de corte permite estimar la rugosidad superficial resultante, pero de una manera menos precisa que utilizando el espesor de viruta.

- El análisis aislado de las velocidades de avance y rotación no son adecuados para analizar la rugosidad, ya que deben tratarse una respecto de la otra. Para facilitar la comprensión de los resultados se debe utilizar el espesor de viruta, ya que integra los principales parámetros que intervienen en el proceso y que demuestran tener un claro efecto tanto sobre la rugosidad superficial como sobre la potencia de corte. 
BOSQUE 25(3): 25-32, 2004

Efecto del cambio de las condiciones de maquinado sobre la rugosidad superficial de Pinus radiata D. Don

\section{AGRADECIMIENTOS}

El presente estudio fue realizado gracias al apoyo financiero proporcionado por la Dirección de Investigación y Desarrollo (D.I.D.) de la Universidad Austral de Chile, por intermedio del Proyecto DID S-2002-04 "Contribución al maquinado de especies madereras de interés económico para la industria del mueble. Estudio de los procesos de corte y la calidad en diferentes condiciones de maquinado".

\section{BIBLIOGRAFIA}

(1) LEMASTER R. L., D. A. DORNFELD. Measurement of surface quality of sawn and planed surfaces with laser. Proceedings of the $7^{\text {th }}$ Wood Machining Seminar. Richmond: University of California. October 18-20. 1982, p. 54-61.

(2) AGUILERA A., P. J. MEAUSOONE, P. MARTIN Optimización de las condiciones de corte en el maquinado de madera. Estimación del desgaste del elemento de corte en fresado. Bosque (Chile), 2000, vol. $21 \mathrm{~N}^{\circ} 2$ p. 127-135.

(3) AGUilerA A., P. J. MEAUSOONE, P. MARTIN. Wood material influence in routing operations: the MDF case. Holz als Roh-und Werkstoff, 2000, vol. 58, No 4, p. 278-283.

(4) AGUILERA A., P. J. MEAUSOONE, P. MARTIN. Estimation de l'usure en défonçage par la mesure de l'évolution du rapport effort tangentiel/effort normal Proceedings of the $14^{\text {th }}$ International Wood Machining Seminar. Paris: Epinal, Cluny. 12-19 September 1999 p. 19-29.

(5) LEMASTER R. L., L. LU, S. JACKSON. The use of process monitoring techniques on a CNC wood router. Part 2. Use of a vibration accelerometer to monitor too wear and workpiece quality. Forest Prod. J., 2000, vol. $50, \mathrm{~N}^{\circ} 9$, p. $59-64$

(6) KIVIMAA E Cutting force in woodworking. Finland: The State Institute for Technical Research. 1950. Publication 18. p. 102

(7) KOCH P. Wood machining processes. A volume in the Wood Processing Series. New York: The Ronald Press Company, 1964, 529 p.

(8) CYRA G, C TANAKA. The effects of wood-fiber directions on acoustic emission in routing. Wood Science and Technology, 2000, vol. 34, p. 237-252. 